\title{
ON CONTRACTIONS SIMILAR TO ISOMETRIES AND TOEPLITZ OPERATORS
}

\author{
BÉLA SZ.-NAGY and CIPRIAN FOIAS,
}

\section{Preliminaries and introduction}

1. The (unitarily equivalent) canonical model of a completely nonunitary contraction $T$ on a (separable, complex) Hilbert space is the operator $S(\Theta)$ on the space $\mathfrak{S}(\Theta)$, associated with a purely contractive analytic function $\left\{\mathbb{E}, \mathfrak{E}_{*}, \Theta(\lambda)\right\}^{1}$ in the following manner ${ }^{2}$

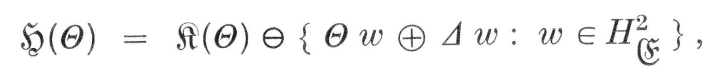

where

$$
\Re(\Theta)=H_{\mathbb{E}_{*}}^{2} \oplus \overline{\Delta L_{\mathfrak{E}}^{2}}, \quad \Delta\left(e^{i t}\right)=\left[I-\Theta\left(e^{i t}\right) * \Theta\left(e^{i t}\right)\right]^{1 / 2},
$$

and

$$
S(\Theta)(u \oplus v)=P_{\mathfrak{S}(\Theta)}(\chi u \oplus \chi v), \quad u \oplus v \in \mathfrak{S}(\Theta),
$$

$\chi$ denoting the function $\chi(\lambda) \equiv \lambda$; cf. [4], Chapter VI.

We have $\mathfrak{H}(\Theta)=\{0\}$ if and only if both $\mathbb{E}$ and $\mathfrak{E}_{*}$ are zero (i.e. equal $\{0\})$; cf. [4], Proposition VI.3.2. On the other hand, $\mathfrak{\mathscr { C }}(\Theta)=\mathfrak{\Re}(\Theta)$

${ }^{1}$ We denote by $\{\mathfrak{A}, \mathfrak{B}, \Phi(\lambda)\}$ an analytic function on the unit disc, whose values are operators from the Hilbert space $\mathfrak{A}$ into the Hilbert space $\mathfrak{B}$, both spaces being supposed complex and separable. This function is bounded if $\|\Phi(\cdot)\|_{\infty}=$ $\sup _{\lambda}\|\Phi(\lambda)\|$ is finite; it is contractive if $\|\Phi(\lambda)\| \leqq 1$, and purely contractive if, moreover, $\|\Phi(0) a\|<\|a\|$ for all $a \in \mathfrak{U}, a \neq 0$. For a bounded analytic function the radial limits $\Phi\left(e^{i t}\right)=\lim \Phi\left(r e^{i t}\right)(r \rightarrow 1-0)$ exist in the strong sense, almost everywhere on the unit circle.

${ }^{2} L_{\mathfrak{F}}^{2}$ denotes the Hilbert space of $\mathbb{E}$-vector valued, norm-square integrable functions on the unit circle, with respect to normalized Lebesgue measure. $H_{\mathbb{E}}^{2}$ is its subspace of functions $u\left(e^{i t}\right) \sim \sum_{k=0}^{\infty} a_{k} e^{i k t}$; these are radial limits a.e. of the corresponding analytic functions $u(\lambda)=\sum_{k=0}^{\infty} a_{k} \lambda^{k}$ in the unit disc. 
if and only if $\Theta w \oplus \Delta w=0$, i.e. $w=0$ for all $w \in H_{⿷ 匚 E}^{2}$, that is, if (F) is zero. Thus the inequalities $0 \neq \mathfrak{H}(\Theta) \neq \mathfrak{N}(\Theta)$ simultaneously hold if and only if

$$
\text { (F) } \neq\{0\} \text {. }
$$

We shall assume (1.4) in the sequel.

The assumption that $\Theta(\lambda)$ be purely contractive has the effect that the operator $V(\Theta)$ defined on $\Re(\Theta)$ by

$$
V(\Theta)(u \oplus v)=\chi u \oplus \chi v, \quad u \oplus v \in \mathcal{K}(\Theta),
$$

is the minimal isometric dilation of $S(\Theta)$.

Note that (1.2) is just the Wold decomposition of the space $\mathfrak{N}(\Theta)$ generated by the isometry $V(\Theta)$, that is,

$$
\bigcap_{n \geqq 0} V(\Theta)^{n} \Re(\Theta)=\{0\} \oplus \overline{\Delta L_{\mathfrak{E}}^{2}} \cdot
$$

2. In any space $L^{2}$ of scalar or vector valued functions $u$ on the unit circle we denote by

$$
[u]_{+} \text {and }[u]_{-}
$$

the orthogonal projections of $u$ to the subspaces

$$
H^{2} \text { and } L^{2} \ominus H^{2} \text {, }
$$

respectively.

With a bounded analytic function $\{\mathfrak{A}, \mathfrak{B}, \Phi(\lambda)\}$ we associate the operator

$$
T(\Phi): H_{\mathfrak{Y}}^{2} \rightarrow H_{\mathfrak{B}}^{2}
$$

defined by

$$
(T(\Phi) u)\left(e^{i t}\right)=\left[\Phi\left(e^{-i t}\right) u\left(e^{i t}\right)\right]_{+} ;
$$

such operators are also called co-analytic Toeplitz operators.

Observe that if $W$ is the canonical unitary transformation $W: H^{2} \rightarrow$ $L^{2} \ominus H^{2}$ defined by

$$
W: u\left(e^{i t}\right) \mapsto e^{-i t} u\left(e^{-i t}\right) \quad\left(u \in H^{2}\right),
$$

then the transformed operator

$$
T^{\wedge}(\Phi)=W_{\mathfrak{B}} T(\Phi) W_{\mathfrak{A}}^{-1}: \quad L_{\mathfrak{A}}^{2} \ominus H_{\mathfrak{A}}^{2} \rightarrow L_{\mathfrak{B}}^{2} \ominus H_{\mathfrak{B}}^{2}
$$

is given by

$$
\left(T^{\wedge}(\Phi) \varphi\right)\left(e^{i t}\right)=\left[\Phi\left(e^{i t}\right) \varphi\left(e^{i t}\right)\right]_{-} \quad\left(\varphi \in L_{\mathfrak{Q}}^{2} \ominus H_{\mathfrak{A}}^{2}\right) .
$$


3. The principal aim of this paper is to derive a condition for an operator valued contractive analytic function $\left\{\mathbb{E}, \mathbb{E}_{*}, \Theta(\lambda)\right\}$ in the unit disc to admit a "left-inverse", i.e. an operator valued bounded analytic function $\left\{\mathbb{E}_{*}, \mathbb{E}, D(\lambda)\right\}$ such that

$$
D(\lambda) \Theta(\lambda)=I_{[5},
$$

and estimates for $\|D(\cdot)\|_{\infty}$. This condition will involve the operator $T(\Theta)$, or equivalently, its unitary transform $T^{\wedge}(\Theta)$.

The theorem obtained reduces in the particular case when $\Theta(\lambda)$ is a finite column vector over $H^{\infty}$ to a recent result of Arveson [1], but it gives even in this case better estimates.

\section{A general condition implying similarity}

Proposition 1. Suppose $T$ is a contraction on a Hilbert space $\mathfrak{H}=\mathfrak{S}^{\prime} \oplus \mathfrak{S}^{\prime \prime}$ such that

(i) the subspace $\mathfrak{S C}^{\prime}$ is invariant for $T$ and $T \mid \breve{S}^{\prime}$ is isometric,

(ii) $\inf \left\{\lim _{n \rightarrow \infty}\left\|T^{n} h\right\|: h \in \mathfrak{S}^{\prime \prime},\|h\|=1\right\}=\eta>0$.

Then there exists an invertible operator $X$ from $\mathfrak{H}$ onto some Hilbert space $\Omega$ such that $X T X^{-1}$ is an isometry on $\mathbb{\Omega}$ and

$$
\|X\|\left\|X^{-1}\right\| \leqq 1 / \eta
$$

Proof. Let $A=\left(\lim _{n \rightarrow \infty} T^{* n} T^{n}\right)^{1 / 2}$; the strong limit exists because $T$ is a contraction. We have $T^{*} A^{2} T=A^{2}$, and therefore $\|A T h\|=$ $\|A h\|$ for all $h \in \mathfrak{H}$. Thus there exists an isometry $Z$ on $\mathbb{L}=\overline{A \mathscr{H}}$ such that

$$
A T=Z A
$$

For $\quad h^{\prime} \in \mathfrak{H}^{\prime} \quad$ we have, by (i), $\quad\left\|T^{n} h^{\prime}\right\|=\left\|h^{\prime}\right\|(n=0,1, \ldots)$. Hence,

$$
\left\|A h^{\prime}\right\|^{2}=\left(A^{2} h^{\prime}, h^{\prime}\right)=\lim _{n}\left\|T^{n} h^{\prime}\right\|^{2}=\left\|h^{\prime}\right\|^{2} .
$$

As $0 \leqq A \leqq I$, equality $\left\|A h^{\prime}\right\|=\left\|h^{\prime}\right\|$ implies $A h^{\prime}=h^{\prime}$. Therefore $\mathfrak{H}^{\prime}$ is invariant for $A$, and hence so is $\mathfrak{S}^{\prime \prime}$ : the decomposition $\mathfrak{\mathscr { C }}=$ $\mathfrak{S}^{\prime} \oplus \mathfrak{S}^{\prime \prime}$ is reducing for $A$. Clearly,

$$
\eta=\inf \left\{\left\|A h^{\prime \prime}\right\|: h^{\prime \prime} \in \mathscr{\mathfrak { S }}^{\prime \prime},\left\|h^{\prime \prime}\right\|=1\right\} .
$$

Because $\quad\left\|A\left(h^{\prime}+h^{\prime \prime}\right)\right\|^{2}=\left\|h^{\prime}+A h^{\prime \prime}\right\|^{2}=\left\|h^{\prime}\right\|^{2}+\left\|A h^{\prime \prime}\right\|^{2}$ and $0 \leqq$ $\eta \leqq 1$, we infer that

$$
\|A h\| \geqq \eta\|h\| \quad \text { for all } h \in \tilde{5} \text {. }
$$


Denote by $X$ the operator $X: \mathfrak{H C} \rightarrow \mathfrak{L}(=\overline{A \mathfrak{F}})$ induced by $A$. Then, by (2.2), $X T X^{-1}=Z$. Moreover, $\|X\| \leqq 1$, and by (2.3), $\left\|X^{-1}\right\| \leqq 1 / \eta$. Thus (2.1) holds true.

\section{A connection between the operators $S(\Theta)$ and $T(\Theta)$}

Consider the operator $S(\Theta)$ generated by a purely contractive analytic function $\left\{\mathbb{E}_{,} \mathbb{E}_{*}, \Theta(\lambda)\right\}$ as in 1.1, that is, with non-zero $\mathbb{E}$.

First observe that the linear manifold

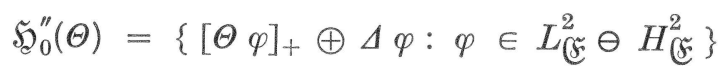

and its closure $\mathfrak{S}^{\prime \prime}(\Theta)$ are contained in $\mathfrak{S}(\Theta)$. Indeed, $\mathfrak{S}_{0}^{\prime \prime}(\Theta)$ is orthogonal to any vector of the form $\Theta w \oplus \Delta w\left(w \in H_{[\xi}^{2}\right)$, because

$$
\begin{aligned}
\left([\Theta \varphi]_{+}, \Theta w\right)+(\Delta \varphi, \Delta w) & =(\Theta \varphi, \Theta w)+(\Delta \varphi, \Delta w) \\
& =\left(\left(\Theta^{*} \Theta+\Delta^{2}\right) \varphi, w\right)=(\varphi, w)=0 .
\end{aligned}
$$

Let $\mathfrak{S g}^{\prime}(\Theta)=\mathfrak{S}(\Theta) \ominus \mathfrak{S g}^{\prime \prime}(\Theta)$. Clearly, we have:

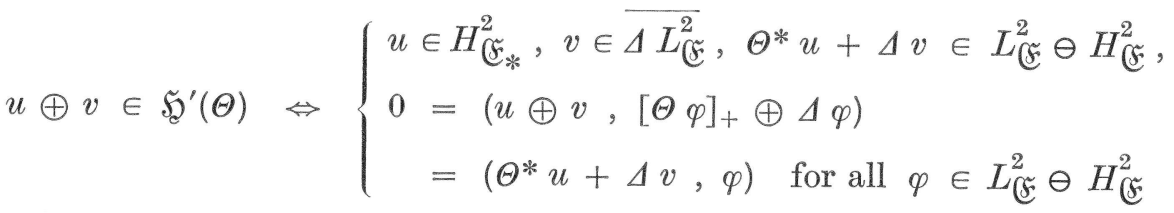

and therefore,

$$
\mathfrak{S}^{\prime}(\Theta)=\left\{u \oplus v \in \mathfrak{S}(\Theta): \Theta^{*} u+\Delta v=0\right\} .
$$

It follows that if $u \oplus v \in \mathfrak{S}^{\prime}(\Theta)$ then $\chi u \oplus \chi v \in \mathfrak{S}^{\prime}(\Theta)$, and hence, $\mathfrak{H}^{\prime}(\Theta)$ is invariant for $S(\Theta)$; moreover, $S(\Theta) \mid \mathfrak{S g}^{\prime}(\Theta)$ is an isometry, namely multiplication by $\chi$.

A straightforward computation shows that for any $u \oplus v \in \mathfrak{K}(\Theta)$ (cf. (1.2)) its projection to $\mathfrak{\pi}(\Theta) \ominus \mathfrak{S}(\Theta)$ equals

$$
\Theta w \oplus \Delta w \text {, where } w=[x]_{+}, x=\Theta^{*} u+\Delta v \text {. }
$$

Therefore,

$$
\left\|P_{\mathfrak{S}(\Theta)}(u \oplus v)\right\|^{2}=\|u \oplus v\|^{2}-\|\Theta w \oplus \Delta w\|^{2}=\|u \oplus v\|^{2}-\|w\|^{2} .
$$

Apply this to $\chi^{n} u \oplus \chi^{n} v(n=0,1,2, \ldots)$ as well, and obtain

$$
\lim _{n \rightarrow \infty}\left\|P_{\mathfrak{S}(\Theta)}\left(\chi^{n} u \oplus \chi^{n} v\right)\right\|^{2}=\|u \oplus v\|^{2}-\lim _{n \rightarrow \infty}\left\|\left[\chi^{n} x\right]_{+}\right\|^{2}
$$


the last limit obviously equals $\|x\|^{2}$. Thus we have for $u \oplus v \in \mathfrak{S}(\Theta)$ :

$$
\lim _{n \rightarrow \infty}\left\|S(\Theta)^{n}(u \oplus v)\right\|^{2}=\|u \oplus v\|^{2}-\left\|\Theta^{*} u+\Delta v\right\|^{2} .
$$

Let, in particular, $h=u \oplus v \in \mathfrak{S}_{0}^{\prime \prime}(\Theta)$, say

$$
h=[\Theta \varphi]_{+} \oplus \Delta \varphi, \quad \varphi \in L_{\mathbb{E}}^{2} \ominus H_{⿷ 匚 ⿳}^{2},
$$

then $\Theta^{*}[\Theta \varphi]_{+} \oplus \Delta \Delta \varphi=\left(\Theta^{*} \Theta+\Delta^{2}\right) \varphi-\Theta^{*}[\Theta \varphi]_{-}=\varphi-\Theta^{*}[\Theta \varphi]_{-}$; hence,

$$
\begin{aligned}
\lim _{n \rightarrow \infty}\left\|S(\Theta)^{n} h\right\|^{2} & =\|h\|^{2}-\left\|\varphi-\Theta^{*}[\Theta \varphi]-\right\|^{2} \\
& =\|h\|^{2}-\|\varphi-B \varphi\|^{2},
\end{aligned}
$$

where $B$ denotes the operator on $L_{\mathbb{E}}^{2} \ominus H_{\mathbb{E}}^{2}$ defined by

$$
B \varphi=\Theta^{*}[\Theta \varphi]
$$

$B$ is selfadjoint and $0 \leqq B \leqq I$; indeed, we have

$$
(B \varphi, \varphi)=\left([\Theta \varphi]_{-}, \Theta \varphi\right)=\left([\Theta \varphi]_{-},[\Theta \varphi]_{-}\right)=\left\|T^{\wedge}(\Theta) \varphi\right\|^{2},
$$

where $T^{\wedge}(\Theta)$ is the transformed Toeplitz operator defined by (1.8). We have

$$
\begin{aligned}
\|h\|^{2} & =\left\|[\Theta \varphi]_{+}\right\|^{2}+\|\Delta \varphi\|^{2}=\left(\|\Theta \varphi\|^{2}-\|[\Theta \varphi]-\|^{2}\right)+\|\Delta \varphi\|^{2} \\
& =\|\varphi\|^{2}-\|[\Theta \varphi]-\|^{2}=\|\varphi\|^{2}-(B \varphi, \varphi)=\|C \varphi\|^{2},
\end{aligned}
$$

where $C=(I-B)^{1 / 2}$, and

$$
\begin{aligned}
\|h\|^{2}-\|\varphi-B \varphi\|^{2} & =\|\varphi\|^{2}-(B \varphi, \varphi)-\|\varphi\|^{2}+2(B \varphi, \varphi)-\|B \varphi\|^{2} \\
& =(B \varphi, \varphi)-(B \varphi, B \varphi)=(B C \varphi, C \varphi) .
\end{aligned}
$$

From (3.3), (3.5) and (3.6) we infer that the infima

$$
\inf _{h}\left\{\lim _{n \rightarrow \infty}\left\|S(\Theta)^{n} h\right\|: h \in \mathfrak{H}_{0}^{\prime \prime}(\Theta),\|h\|=1\right\}
$$

and

$$
\inf _{\psi}\{(B \psi, \psi): \psi \in \text { range } C,\|\psi\|=1\}
$$

are equal. They remain, by continuity, unchanged and therefore equal to each other if we allow $h$ and $\psi$ to run over all unit vectors in $\mathfrak{H}^{\prime \prime}(\Theta)$ and in the closure $\Re(C)$ of the range of $C$, respectively. Now $\Re(C)$ is obviously reducing $B$ and for $\psi$ in the orthogonal complement of $\Re(C)$ we have $C \psi=0, B \psi=\psi, \quad(B \psi, \psi)=\|\psi\|^{2}$. Hence we infer that the second infimum does not change even if we allow $\psi$ to run over all unit vectors in $L_{\mathfrak{E}}^{2} \ominus H_{\mathfrak{S}}^{2}$. 
Recalling (3.4) and observing that the Toeplitz operator $T(\Theta)$ clearly has the same lower bound as its unitary transform $T^{\wedge}(\Theta)$, on the respective unit spheres, we conclude:

Proposition 2. For any purely contractive analytic $\left\{\mathbb{E}, \mathbb{E}_{*}, \Theta(\lambda)\right\}$ the decomposition $\mathfrak{S}(\Theta)=\mathfrak{S E}^{\prime}(\Theta) \oplus \mathfrak{F}^{\prime \prime}(\Theta)$ of the space $\mathfrak{S}(\Theta)$ defined by (3.1) and (3.2) is such that

(i) $S(\Theta) \mid \mathfrak{S}^{\prime}(\Theta)$ is an isometry on $\mathfrak{S g}^{\prime}(\Theta)$,

(ii) The infima

$$
\inf _{h}\left\{\lim _{n \rightarrow \infty}\left\|S(\Theta)^{n} h\right\|: h \in \mathfrak{S}^{\prime \prime}(\Theta),\|h\|=1\right\}
$$

and

$$
\inf _{u}\left\{\|T(\Theta) u\|: u \in H_{⿷ 匚 ⿳}^{2},\|u\|=1\right\}
$$

are equal to the same value $\eta=\eta(\Theta)$.

\section{Similarity of $S(\Theta)$ to an isometry}

In case the quantity $\eta=\eta(\Theta)$ defined in Proposition 2 is non-zero we can apply Proposition 1 and conclude that $S(\Theta)$ is similar to some isometry $Z$ on a space $\mathbb{Q}$, i.e. there exist operators

$$
X: \quad \mathfrak{H}(\Theta) \rightarrow \mathfrak{R}, \quad X^{\prime}: \quad \mathfrak{L} \rightarrow \mathfrak{H}(\Theta)
$$

such that

$$
Z X=X S(\Theta), \quad S(\Theta) X^{\prime}=X^{\prime} Z, \quad X^{\prime}=X^{-1}
$$

and moreover,

$$
\left\|X^{\prime}\right\|\|X\| \leqq 1 / \eta
$$

Now the following is true:

Proposition 3. From (4.1), $Z$ an isometry, it follows that there exists a bounded analytic function $\left\{\mathbb{E}_{*}, \mathbb{E}, D(\lambda)\right\}$ such that

$$
D(\lambda) \Theta(\lambda)=I_{\mathbb{E}}, \quad\|D(\cdot)\|_{\infty} \leqq\left\|X^{\prime}\right\|\|X\| .
$$

Proof. The existence of a bounded analytic $D(\lambda)$ with the property $D(\lambda) \Theta(\lambda)=I$ is proved in Theorem 2.4 of $[5]$, and an estimate for $\|D(\cdot)\|_{\infty}$ can also be deduced from the proof of that theorem. For convenience, we give a direct and complete proof.

This proof is based upon the "commutant lifting theorem" of [3]; see also [4], Sec.II.2.3. Since $Z$ is its own minimal isometric dilation this theorem asserts in this case that there exist operators 


$$
Y: \quad \mathfrak{N}(\Theta) \rightarrow \Omega, \quad Y^{\prime}: \quad \mathfrak{L} \rightarrow \mathfrak{N}(\Theta)
$$

such that (using the notations of Sec. 1.1):

$$
\begin{gathered}
Z Y=Y V(\Theta), \quad V(\Theta) Y^{\prime}=Y^{\prime} Z, \\
Y P_{\mathfrak{H}(\Theta)^{\perp}}=0,{ }^{3} \\
X=Y \mid \mathfrak{H}(\Theta), \quad X^{\prime}=P_{\mathfrak{S}(\Theta)} Y^{\prime},
\end{gathered}
$$

and

$$
\|Y\|=\|X\|, \quad\left\|Y^{\prime}\right\|=\left\|X^{\prime}\right\| .
$$

Moreover, $X^{\prime} X=I_{\mathfrak{H}(\Theta)}$ implies by $(4.5)$ :

$$
P_{\mathfrak{H}(\Theta)} Y^{\prime} Y \mid \mathfrak{S}(\Theta)=I_{\mathfrak{H}(\Theta)} ;
$$

on account of (4.4) this is equivalent to the condition

$$
P_{\mathfrak{H}(\Theta)}\left(I-Y^{\prime} Y\right) k=0 \quad \text { for all } k \in \mathfrak{H}(\Theta) \text {. }
$$

From (4.4) and (4.7) it easily follows that the operator

$$
F=I-Y^{\prime} Y
$$

satisfies the conditions

$$
F^{2}=F \quad \text { and } \quad F \mathfrak{H}(\Theta)=\mathscr{H}(\Theta)^{\perp} .
$$

Thus $F$ is a (bounded) parallel projection of $\mathfrak{R}(\Theta)$ onto $\mathfrak{S}(\Theta)^{\perp}$.

Observe that

$$
\omega: w \mapsto \Theta w \oplus \Delta w \quad\left(w \in H_{\mathbb{E}}^{2}\right)
$$

is a unitary operator $\omega: H_{(\mathfrak{E})}^{2} \rightarrow \mathfrak{H}(\Theta)^{\perp}$, which commutes with multiplication by the scalar function $\chi$. As (4.3) implies $F V(\Theta)=V(\Theta) F$, the operator $F$ also commutes with multiplication by $\chi$. As a consequence we have

$$
\begin{aligned}
F \cap V(\Theta)^{n} \mathfrak{K}(\Theta) & \subset \underset{n \geqq 0}{\cap} V(\Theta)^{n} F \mathfrak{K}(\Theta)=\bigcap_{n \geqq 0} V(\Theta)^{n} \mathfrak{S}(\Theta)^{\perp}=\bigcap_{n \geqq 0} \chi^{n} \cdot \omega H_{\mathfrak{C}}^{2} \\
& =\omega \bigcap_{n \geqq 0} \chi^{n} H_{\mathbb{E}}^{2}=\omega\{0\}=\{0\},
\end{aligned}
$$

thus by (1.6)

$$
F(0 \oplus v)=0 \quad \text { for any } v \in \overline{\Delta L_{\mathbb{E}}^{2}} \text {. }
$$

Combining this with (4.9) we get in particular

$$
{ }^{3} \tilde{\mathfrak{S}(\Theta) \perp}=\mathfrak{N}(\Theta) \Theta \mathfrak{H}(\Theta)=\left\{\Theta w \oplus \Delta w: w \in H_{[\mathfrak{L}}^{2}\right\} ; \text { cf. (1.1). }
$$




$$
\begin{aligned}
\Theta u \oplus \Delta u & =F(\Theta u \oplus \Delta u) \\
& =F(\Theta u \oplus 0)+F(0 \oplus \Delta u)=F(\Theta u \oplus 0)
\end{aligned}
$$

for any $u \in H_{\mathbb{E}}^{2}$.

Applying (4.9) again, we see that for every $k \in \mathfrak{N}(\Theta)$ there exists a unique $w \in H_{⿷ 匚}^{2}$ such that $F k=\omega w$. Choosing in particular $k=u_{*} \oplus 0$, $u_{*} \in H_{\mathbb{E}_{*}}^{2}$, equation

$$
F\left(u_{*} \oplus 0\right)=\omega \cdot D u_{*}
$$

defines an operator $D: H_{\mathbb{E}_{*}}^{2} \rightarrow H_{\mathbb{E}}^{2}$; clearly

$$
\|D\| \leqq\|F\| \text {. }
$$

For $F$, the inequality $\|F\| \leqq 1+\left\|Y^{\prime}\right\|\|Y\|$ is immediate from the definition (4.8). But we have even

$$
\|F\|=\|I-F\|,
$$

and hence the inequality ${ }^{4}$

$$
\|F\| \leqq\left\|Y^{\prime}\right\|\|Y\|
$$

Indeed, (4.13) holds for any (bounded) parallel projection of a Hilbert space onto a non-trivial subspace. This follows, namely, from the relation ${ }^{5}$

$$
\left\|F^{\prime}\right\|^{-2}=1-\sup |(h, g)|^{2}
$$

where $h, g$ run over the sets of unit vectors satisfying $(I-F) h=0$ and $F g=0$, respectively, and from the symmetry of this relation in $F$ and $I-F$. Note that in the case under consideration $F$ projects indeed to a non-trivial subspace of $\Re(\Theta)$, because our assumption $\mathbb{E} \neq\{0\}$ assures that $\{0\} \neq \mathscr{\mathfrak { S }}(\Theta) \neq \mathscr{I}(\Theta)$.

Thus, taking account of (4.6) and (4.12), we have

$$
\|D\| \leqq\left\|X^{\prime}\right\|\|X\|
$$

Next observe that as $F$ and $\omega$ commute with multiplication by $\chi$ so does $D$ too. Hence it follows (ef. Lemma V.3.1 in [4]) that $D$ itself is a multiplication operator, viz.

$$
\left(D u_{*}\right)(\lambda)=D(\lambda) u_{*}(\lambda)
$$

\footnotetext{
4 The authors are indebted for this ingenious and useful remark to Professor T. Ando from Sapporo (Japan), presently visiting Szeged (Hungary).

5 This formula for $F$ is due to $\mathrm{V}$. E. Ljance and is reproduced in the book of I. C. Gohberg and M. G. KreĬn [2], VI.5.4.
} 
where $\left\{\mathfrak{E}_{*}, \mathbb{E}, D(\lambda)\right\}$ is a bounded analytic function,

$$
\|D(\cdot)\|_{\infty}=\|D\| .
$$

On account of definition (4.11) of $D$ we have in particular $F(\Theta u \oplus 0)=\omega \cdot D \Theta u$ for any $u \in H_{[⿷ 匚 ⿳}^{2}$, while (4.10) means $F(\Theta u \oplus 0)=\omega \cdot u$. Therefore, $u=D \Theta u$, and hence

$$
D(\lambda) \Theta(\lambda)=I_{[⿷} \text {. }
$$

Recalling (4.15) and (4.16) the proof of Proposition 3 is done.

\section{Conclusions}

Combining Propositions $1-3$ we conclude that if $\left\{\left[\mathbb{E}, \mathfrak{F}_{*}, \Theta(\lambda)\right\}\right.$ is a purely contractive analytic function for which ${ }^{6}$

$$
\inf \left\{\|T(\Theta) u\|: u \in H_{⿷ 匚 ⿳}^{2},\|u\|=1\right\}=\eta>0,
$$

then there exists an analytic function $\left\{\mathbb{E}_{*}, \mathbb{E}, D(\lambda)\right\}$ such that

$$
D(\lambda) \Theta(\lambda)=I_{\mathbb{E}} \text { and }\|D(\cdot)\|_{\infty} \leqq 1 / \eta \text {. }
$$

Now it is easy to get rid of the restriction "purely contractive". Indeed, every contractive analytic function $\left\{\mathbb{E}, \mathfrak{E}_{*}, \Theta(\lambda)\right\}$ is, according to Proposition V.2.1 of [4], direct sum of a purely contractive analytic function $\left\{\mathbb{F}^{0}, \mathbb{F}_{*}^{0}, \Theta^{0}(\lambda)\right\}$ and of a unitary valued constant function $\left\{\mathbb{E}^{\prime}, \mathbb{F}_{*}^{\prime}, \Theta^{\prime}\right\}$ $\left(\mathbb{E}=\mathfrak{E}^{0} \oplus \mathbb{E}^{\prime}, \mathfrak{E}_{*}=\mathfrak{F}_{*}^{0} \oplus \mathbb{C}_{*}^{\prime}\right)$. Hence it follows for any $u=$ $u^{0} \oplus u^{\prime} \in H_{\mathbb{C}}^{2}$ with components $u^{0} \in H_{\mathfrak{C}^{2} 0}^{2}, u^{\prime} \in H_{\mathbb{E}^{\prime}}^{2}$ that

$$
T(\Theta) u=T\left(\Theta^{0}\right) u^{0} \oplus \Theta^{\prime} u^{\prime}, \quad\|T(\Theta) u\|^{2}=\left\|T\left(\Theta^{0}\right) u^{0}\right\|^{2}+\left\|u^{\prime}\right\|^{2} .
$$

In the case the first component is missing, but (5.1) is fulfilled, then, necessarily, $\left[5^{\prime} \neq\{0\}\right.$ and $\eta=1$, and a trivial solution for $D(\lambda)$ in (5.2) is the constant function $\left\{\mathbb{E}_{*}^{\prime}, \mathbb{E}^{\prime}, \Theta^{\prime *}\right\}$. If both components are present then $\eta$ equals the analogous quantity $\eta^{0}$ formed for $\Theta^{0}$ (because $\left.\eta^{0} \leqq 1\right)$ and hence (5.1) implies the existence of an analytic function $\left\{\mathfrak{F}_{*}^{0}, \mathfrak{E}^{0}, D^{0}(\lambda)\right\}$ satisfying $D^{0}(\lambda) \Theta^{0}(\lambda)=I_{\mathfrak{F}_{0}^{0}},\left\|D^{0}(\cdot)\right\|_{\infty} \leqq 1 / \eta^{0}=1 / \eta$. Setting $D(\lambda)=D^{0}(\lambda) \oplus \Theta^{*}$ we get a solution for (5.2).

So we can formulate our main result:

$\mathrm{Th}$ e or e m. If the contractive analytic function $\left\{\left[\mathbb{E}, \mathbb{F}_{*}, \Theta(\lambda)\right\}\right.$ is such that

\footnotetext{
${ }^{6}$ This condition obviously implies $[5 \neq\{0\}$.
} 
(*)

$$
\|T(\Theta) u\| \geqq \eta\|u\| \quad \text { for an } \eta>0 \text { and all } u \in H_{\mathbb{E}^{\tilde{E}}}^{2},
$$

then there exists an analytic function $\left\{\mathbb{E}_{*}, \mathfrak{E}, D(\lambda)\right\}$ such that

$$
D(\lambda) \Theta(\lambda)=I_{[} \text {for }|\lambda|<1 \text {, and }\|D(\cdot)\|_{\infty} \leqq 1 / \eta \text {. }
$$

$\mathrm{R}$ e $\mathrm{m}$ a $\mathrm{rk}$ 1. In the special case of a function $\left\{E^{1}, E^{N}, \Theta(\lambda)\right\}$, where

$$
\Theta(\lambda)=\left[\begin{array}{c}
\vartheta_{1}(\lambda) \\
\vdots \\
\vartheta_{N}(\lambda)
\end{array}\right],
$$

the theorem can be given the following from: If

$$
\sum_{k=1}^{N}\left|\vartheta_{k}(\lambda)\right|^{2} \leqq 1 \quad \text { for }|\lambda|<1
$$

and

$$
\sum_{k=1}^{N}\left\|T\left(\vartheta_{k}\right) u\right\|^{2} \geqq \eta^{2}\|u\|^{2} \quad \text { for an } \eta>0 \text { and all } u \in H^{2},
$$

then there exist $d_{k} \in H^{\infty} \quad(k=1, \ldots, N)$ such that, for $|\lambda|<1$,

$$
\sum_{k=1}^{N} d_{k}(\lambda) \vartheta_{k}(\lambda)=1 \text { and }\left|d_{k}(\lambda)\right| \leqq 1 / \eta \quad(k=1, \ldots, N) \text {. }
$$

Observe that if

$$
f_{k} \in H^{\infty},\left\|f_{k}\right\|_{\infty} \leqq 1 \quad(k=1, \ldots, N)
$$

and

$$
\sum_{k=1}^{N}\left\|T\left(f_{k}\right) u\right\|^{2} \geqq \varepsilon^{2}\|u\|^{2} \quad \text { for some } \varepsilon>0 \text { and all } u \in H^{2},
$$

then the functions $\vartheta_{k}(\lambda)=f_{k}(\lambda) / \sqrt{N}(k=1, \ldots, N)$ satisfy the above requirements, with $\eta=\varepsilon / \sqrt{N}$. Hence there exist functions $d_{k}(\lambda)$ as in (5.3), and therefore the functions $g_{k}(\lambda)=d_{k}(\lambda) / \sqrt{N}$ satisfy

$$
\sum_{k=1}^{N} g_{k}(\lambda) f_{k}(\lambda)=1 \text { and }\left\|g_{k}\right\|_{\infty} \leqq 1 / \varepsilon(k=1, \ldots, N) .
$$

The fact that assumptions (5.4) and (5.5) imply the existence of $g_{k} \in H^{\infty}$ satisfying (5.6) was also proved by Arveson [1], Theorem 6.3, however with the estimate $\left\|g_{k}\right\|_{\infty} \leqq 4 N \varepsilon^{-3}$ only.

$\mathrm{R}$ e mark 2. The functions $k_{\mu}(\lambda)=(1-\mu \lambda)^{-1}(|\mu|<1)$ span

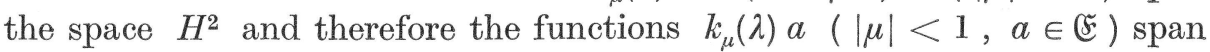
$H_{\mathbb{E}}^{2}$. Thus (*) holds for every $u \in H_{\mathbb{C}}^{2}$ if and only if it holds for the finite linear combinations of these functions. Now observe that 


$$
\left(T(\Theta) k_{\mu} a\right)\left(e^{i t}\right)=\left[\Theta\left(e^{-i t}\right) k_{\mu}\left(e^{i t}\right) a\right]_{+}=k_{\mu}\left(e^{i t}\right) \Theta(\mu) a
$$

and that $\left(k_{\mu}, k_{v}\right)=(1-\bar{v} \mu)^{-1}(|\mu|<1,|v|<1)$. Thus we infer that condition (*) is equivalent to the condition that the kernel

$$
K(\mu, v)=(1-\bar{v} \mu)^{-1}\left(\Theta(v)^{*} \Theta(\mu)-\eta^{2} I\right) \quad(|\mu|<1,|v|<1)
$$

be positive definite, i.e.

$$
\sum_{i=1}^{N} \sum_{j=1}^{N}\left(K\left(\mu_{i}, \mu_{j}\right) a_{j}, a_{i}\right) \geqq 0
$$

for any finite set of points $\mu_{i}$ in the unit disc and vectors $a_{i}$ in $E$.

$\mathrm{R}$ e $\mathrm{mark} 3$. Our Theorem has a rather immediate converse. Indeed if there exists an analytic function $D(\lambda)$ such that

$$
D(\lambda) \Theta(\lambda)=I_{\mathbb{C}} \text { and }\|D(\cdot)\|_{\infty}=1 / \eta<\infty
$$

then we have for $u \in H_{\mathbb{E}}^{2}$

$$
u=[u]_{+}=\left[D\left(e^{-i t}\right) \Theta\left(e^{-i t}\right) u\left(e^{i t}\right)\right]_{+}=\left[D\left(e^{-i t}\right)\left[\Theta\left(e^{-i t}\right) u\left(e^{i t}\right)\right]_{+}\right]_{+},
$$

and hence

$$
\eta^{2}\|u\|^{2} \leqq \eta^{2} \int_{0}^{2 \pi}\left\|D\left(e^{-i t}\right)\left[\Theta\left(e^{-i t}\right) u\left(e^{i t}\right)\right]_{+}\right\|^{2} \frac{d t}{2 \pi} \leqq\|T(\Theta) u\|^{2} .
$$

R e mark 4. From Propositions $1-3$ and Remark 3 it readily results the equality of the infima

$$
\inf \left\{\left\|X^{-1}\right\|\|X\|: X^{-1} S(\Theta) X \text { is an isometry }\right\}
$$

and

$$
\inf \left\{\|D(\cdot)\|_{\infty}: D(\lambda) \Theta(\lambda)=I_{[⿷ 匚 ⿳}\right\}
$$

\section{References}

[1] ARveson, W.: Interpolation problems in nest algebras. - J. Functional Analysis $20,1975,208-233$.

[2] Gohberg, I. C., and M. G. KREIN [И. Ц. Гохберг and М. Г. Крейн] : Введение в теорию линейных несамосопряженных операторов. - Издательство "Наука", Москва, 1965. Translation: Introduction to the theory of linear nonselfadjoint operators. - Translations of Mathematical Monographs 18. American Mathematical Society, Providence, Rhode Island, 1969.

[3] Sz.-NAGY, B., and C. Folas: Dilatation des commutants d'opérateurs. - C. R. Acad. Sci. Paris. Sér. A-B 266, 1968, A493-A495. 
[4] Sz.-NAGY, B., and C. ForAs: Harmonic analysis of operators on Hilbert space. North-Holland Publishing Company, Amsterdam-London / Akadémiai Kiadó, Budapest, 1970.

[5] - - - - On the structure of intertwining operators. - Acta Sci. Math. (Szeged) 35, 1973, 225-256.7

Bolyai Institute

Aradi vértanuk tere 1

H-6720 Szeged

Hungary
Facultatea de Matematica

Str. Academiei 14

Bucharest

Romania

Received 5 September 1975

$7 \mathrm{We}$ use this opportunity to correct some deficiencies of the paper [5].

Page 230: at the end of the 9th row change " $\hat{\Theta}_{1}$ " for " $\hat{\Delta}_{1} "$.

Page 231: insert between the 12th and 13th rows:

$$
"(\delta)_{0} \quad B^{\prime} A_{*}+C^{-1} B=-\hat{\Delta}_{1} D " .
$$

Page 231: 8th row from below, insert " $B^{\prime}=0$ ".

Page 254: papers [6] - [8] in the References have the authors "B. Sz.-NagyC. Foias". 